\title{
Comparison between fasting and non-fasting cut- off values of triglyceride in diagnosing high triglyceride in Chinese hypertensive outpatients
}

\section{Yingying Xie}

Second Xiangya Hospital

\section{Peiliu Qu}

Second Xiangya Hospital

Ling Liu ( $\nabla$ feliuling@csu.edu.cn )

Second Xiangya Hospital

Xiao Du

Second Xiangya Hospital

\section{Shilan Zhang}

Second Xiangya Hospital

\section{Liling Guo}

Second Xiangya Hospital

\section{Qunyan Xiang}

second xiangya hospital

Qiuzhen Lin

Second Xiangya Hospital

Jin Xu

Second Xiangya Hospital

\section{Liyuan Zhu}

Second Xiangya Hospital

\section{Yangrong Tan}

Second Xiangya Hospital

\section{Yan Fu}

second xiangya hospital

\section{Miao Zhang}

Second Xiangya Hospital

\section{Xueyan Zang}

Second Xiangya Hospital

\section{Tie Wen}




\section{Research Article}

Keywords: hypertension, non-fasting, triglyceride, remnant cholesterol, blood lipid test

Posted Date: November 10th, 2021

DOI: https://doi.org/10.21203/rs.3.rs-961584/v2

License: (c) (i) This work is licensed under a Creative Commons Attribution 4.0 International License. Read Full License 


\section{Abstract \\ Background}

Hypertension (HBP) often occurs together with hypertriglyceridemia that indicates elevated triglyceride (TG) and remnant cholesterol (RC) levels. Non-fasting (i.e. postprandial) blood lipid test after a daily meal has been recommended by the European Atherosclerosis Society (EAS). However, little is known about the difference between fasting and non-fasting cut-off values in assessing high TG (HTG) and high RC (HRC) in HBP outpatients.

\section{Methods}

Two hundred and twenty-five Chinese outpatients with HBP were enrolled in this study. According to the time of blood lipid test, they were divided into two groups, i.e. the fasting group $(n=119)$ and the nonfasting group ( $n=139)$. Non-fasting levels of blood lipids at $2 \mathrm{~h}$ after a daily breakfast were also tested in 33 patients among the fasting group. Venous blood samples were collected. Serum levels of blood lipids were measured by the enzymatic and direct methods on a HITACHI 7170A analyzer or estimated via related formulas.

\section{Results}

The non-fasting group had significantly higher levels of TG and RC while lower levels of total cholesterol, low-density lipoprotein cholesterol and non-high-density lipoprotein cholesterol than the fasting group ( $P \otimes 0.05$ ). According to TG and RC cut-off values of the EAS, the percentages of HTG and HRC in the nonfasting group were $67.6 \%$ and $65.6 \%$, respectively, while those in the fasting group were $57.1 \%$ and $52.9 \%$, respectively. However, the percentages of HTG in the fasting state and at $2 \mathrm{~h}$ after a daily breakfast in 33 outpatients did not reach statistical significance (57.6\% v.s. $51.5 \%)$. So did the fasting and at $2 \mathrm{~h}$ nonfasting percentages of HRC in them.

\section{Conclusion}

Non-fasting blood lipid test could find more HBP outpatients with HTG in Chinese outpatients with HBP. However, the percentage of HTG at $2 \mathrm{~h}$ after a daily breakfast seemed to be close to that in the fasting state.

\section{Introduction}

Once hypertension (HBP) coexists with dyslipidemia [1], all-cause mortality and cardiovascular mortality increase sharply [2]. The primary goal of cholesterol-lowering therapy is to decrease low-density lipoprotein cholesterol (LDL-C) to target levels, and the secondary goal is to reduce non-high-density 
lipoprotein cholesterol (non-HDL-C). Non-HDL-C is the total amount of cholesterol contained in lipoproteins other than high-density lipoprotein (HDL). In Chinese patients with HBP, the prevalence of hypertriglyceridemia was significantly higher than that of hypercholesterolemia [3]. Hypertriglyceridemia represents an increase in the amount of triglyceride-rich lipoprotein (TRLs) and their hydrolytic products, remnant lipoproteins (RLPs). Compared with nascent TRLs, RLPs contain more cholesterol ester, have smaller diameters, and thus are regarded as atherogenic as LDL. As a portion of non-HDL-C, remnant lipoprotein cholesterol (i.e. remnant cholesterol, RC) level is positively associated with TG level [4]. Therefore, it is necessary to evaluate the levels of TG and RC in patients with HBP.

Due to the long history of fasting blood lipid test, it is generally accepted by Chinese doctors and patients [5]. Many studies had confirmed a close relationship between fasting LDL-C level and coronary heart disease (CHD) [6, 7]. However, a clinical study with a large population recently shown that non-fasting TG levels at 2 to 4 hours had the strongest association with cardiovascular events rather than fasting TG level [8]. In addition, elevated non-fasting TG, as well as RC levels, were associated with increased risk of myocardial infarction, ischemic heart disease, and death in the general population cohort of Denmark [9]. Non-fasting blood lipid test after a daily meal has been recommended in routine clinical practice by the European Atherosclerosis Society (EAS). This recommendation could be helpful for the diagnosis of hypertriglyceridemia in Chinese outpatients who forget to keep the fasting state.

In this study, we explored the difference in the levels of TG or RC as well as the percentages of high TG (HTG) or high RC (HRC) between the fasting and non-fasting outpatients with HBP. Furthermore, a questionnaire survey was also conducted to learn about the awareness and acceptance of non-fasting blood lipid test in Chinese outpatients with HBP.

\section{Methods}

\subsection{Study subjects}

Two hundred and twenty-five outpatients with primary HBP were enrolled in this study in the Outpatient Department of Cardiovascular Medicine Department, Second Xiangya Hospital, Central South University from July 2019 to March 2021. HBP was defined as a history of systolic blood pressure values $\geq 140 \mathrm{~mm}$ $\mathrm{Hg}$ and/or diastolic blood pressure values $\geq 90 \mathrm{~mm} \mathrm{Hg}$ for at least 3 days [10, 11].

According to the fasting state or not, there were 119 fasting patients and 106 non-fasting ones visiting the Outpatient Department. Thirty-three fasting ones among 119 fasting patients also accepted nonfasting blood lipid test at $2 \mathrm{~h}$ after a daily breakfast. Thus, there were two groups according to the time of blood lipid test, i.e. the fasting group $(n=119)$ and the non-fasting group $(n=139)$. Among all patients, 97 ones accepted a questionnaire survey to learn about the awareness and acceptance of non-fasting blood lipid test. Fasting patients were those with a fasting period of at least $8 \mathrm{~h}$ before blood lipid test. Nonfasting ones were those with blood lipid test within $4 \mathrm{~h}$ after the last meal. 
All of them were excluded from a history of secondary HBP, poorly controlled diabetes, thyroid diseases, liver and kidney diseases, autoimmune diseases, mental diseases, cancer or other severe medical diseases before getting involved. No one took oral hypolipidemic agents or other drugs that affected lipid metabolism. All patients continued to take antihypertensive drugs as usual and their blood pressure was monitored throughout the course. This study was approved by the Ethics Committee of the Second Xiangya Hospital of Central South University and informed consent was obtained from all participants.

\subsection{Specimen Collection}

After at least $8 \mathrm{~h}$ of overnight fasting, venous blood samples were collected in 119 fasting outpatients with HBP. Moreover, venous blood samples were also collected at $2 \mathrm{~h}$ after a daily breakfast in 33 ones among 119 fasting outpatients according to their daily habits. One hundred and six non-fasting outpatients accepted non-fasting blood lipid test after a daily breakfast or lunch, and venous blood samples were collected within $4 \mathrm{~h}$ after a daily meal according to their daily habits.

\subsection{Laboratory assays}

The serum was separated from the venous blood samples. Serum levels of TG and total cholesterol (TC) were measured by automated enzymatic assays, and those of LDL-C and high-density lipoprotein cholesterol (HDL-C) were determined by the direct method, on a HITACHI 7170A analyzer (Instrument Hitachi Ltd., Tokyo, Japan) by a laboratory technician who had no knowledge of this study. The detection kits were provided by Japan and Pure Pharmaceutical Industry Co. (Wako). Levels of RC and non-HDL-C were estimated by the following formulas, $R C=T C-(H D L-C)-(L D L-C)$ and non-HDL-C $=T C-(H D L-C)$, respectively.

\subsection{Cut-off values to determine HTG and HRC}

According to the European joint consensus statement from the EAS [12], the fasting cut-off values to determine HTG and HRC are TG level $1.7 \mathrm{mmol} / \mathrm{L}$ and RC level $0.8 \mathrm{mmol} / \mathrm{L}$, respectively. Their corresponding non-fasting cut-off values to determine $H T G$ and HRC are TG level $2.0 \mathrm{mmol} / \mathrm{L}$ and RC level $0.9 \mathrm{mmol} / \mathrm{L}$, respectively.

Recently, we reported that the non-fasting cut-off values to determine HTG and HRC were TG level 2.02 $\mathrm{mmol} / \mathrm{L}$ [13] and RC level $0.87 \mathrm{mmol} / \mathrm{L}$ [14], respectively, in Chinese subjects.

In this study, the non-fasting cut-off values to determine HTG and HRC recommended by EAS and reported in Chinese subjects were all selected to evaluate the percentages of HTG and HRC in HBP outpatients.

\subsection{Questionnaire survey}

In order to learn about the awareness and acceptance of non-fasting blood lipid test in outpatients with HBP, ninety-seven ones were invited to answer two questions. Each question is a single-choice one. Awareness survey referred to whether outpatients should keep fasting or non-fasting state for blood lipid test. There were four options, i.e. 'fasting', 'non-fasting, 'either is OK' and 'having no idea'. Acceptance 
survey referred to the investigation on the choice made by outpatients again after being told that both fasting and non-fasting lipid test were available and recommended. There were four options, i.e. 'fasting', 'non-fasting', 'either is OK' and 'depending on doctor'.

\subsection{Statistical analysis}

Quantitative variables of normal distribution were expressed as mean \pm standard deviation (SD), and those of non-normal distribution were analyzed statistically after logarithmic conversion. Qualitative variables were expressed as numbers and percentages. The difference of the inter-group quantitative variables was analyzed by independent-samples $t$-test. Paired $t$-test was used for comparing quantitative variables between the two time points. Qualitative variables were compared using Chi-square test for $\mathrm{R} x$ C. All statistical analyses were performed with SPSS version 26.0. All $P$ values were 2-tailed, and $P<0.05$ was considered statistically significant.

\section{Results}

\subsection{General clinical characteristics of two groups}

There was no significant difference in age, gender, body mass index (BMI), waist, systolic or diastolic blood pressure, heart rate, percentage of central obesity and proportion of patients with CHD and diabetes mellitus (DM). The number of current smokers in the non-fasting group was significantly lower than that in the fasting group $(P<0.05$, Table 1$)$. 
Table 1

Comparison of clinical characteristics between two groups

\begin{tabular}{|c|c|c|}
\hline & $\begin{array}{l}\text { Fasting group } \\
(n=119)\end{array}$ & $\begin{array}{l}\text { Postprandial group } \\
(n=139)\end{array}$ \\
\hline Age $(y)$ & $45.5 \pm 10.6$ & $45.8 \pm 9.3$ \\
\hline Men, n (\%) & $80(67.2)$ & $91(65.5)$ \\
\hline BMI $\left(\mathrm{kg} / \mathrm{m}^{2}\right)$ & $27.1 \pm 3.7$ & $26.4 \pm 3.8$ \\
\hline Waist (cm) & $94.1 \pm 10.4$ & $94.3 \pm 11.3$ \\
\hline Current smoking, n (\%) & $32(26.9)$ & $17(2.9)$ * \\
\hline Central obesity, n (\%) & $88(74.0)$ & $97(69.8)$ \\
\hline CHD, n (\%) & $5(4.2)$ & $3(2.7)$ \\
\hline DM, n (\%) & $6(5.0)$ & $6(4.3)$ \\
\hline $\mathrm{SBP}(\mathrm{mm} \mathrm{Hg})$ & $157.2 \pm 22.5$ & $154.6 \pm 20.0$ \\
\hline $\mathrm{DBP}(\mathrm{mm} \mathrm{Hg})$ & $98.1 \pm 15.8$ & $101.0 \pm 77.7$ \\
\hline $\mathrm{HR}(\mathrm{bpm})$ & $85.3 \pm 14.7$ & $88.6 \pm 15.6$ \\
\hline
\end{tabular}

\subsection{Comparison of blood lipid levels between two groups}

Among 106 non-fasting outpatients, 42 ones visited the doctors after breakfast while 64 ones visited the doctors after lunch. Among 106 ones, 62 ones were tested at $2 \mathrm{~h}, 18$ ones were tested at $3 \mathrm{~h}$ and 26 ones were tested at $4 \mathrm{~h}$ after a daily breakfast or lunch. Among 62 ones who were tested at $2 \mathrm{~h}, 28$ ones were tested after breakfast while 34 ones were tested after lunch. Among 18 ones who were tested at $3 \mathrm{~h}, 7$ ones were tested after breakfast while 11 ones were tested after lunch. Among 26 ones who were tested at $4 \mathrm{~h}, 12$ ones were tested after breakfast while 14 ones were tested after lunch (Fig. S1). Compared with the fasting group, the non-fasting group had significantly higher TG and RC levels $(P<0.05)$ while lower levels of TC, LDL-C and non-HDL-C $(P<0.05)$. There was no significant difference in HDL-C level between the two groups (Fig. 1A-F).

\subsection{Comparisons of percentages of HTG and HRC between two groups}

According to the cut-off values of HTG and HRC recommended by the EAS [12], the percentages of HTG and $\mathrm{HRC}$ in the fasting group were $57.1 \%$ and $52.9 \%$, respectively, while those in the non-fasting group 
were $67.6 \%$ and $65.6 \%$, respectively. According to the non-fasting cut-off values to determine HTG and HRC reported in Chinese subjects $[13,14]$, the percentages of HTG and HRC in the non-fasting group were $67.6 \%$ and $67.6 \%$, respectively (Fig. 2A-B).

\subsection{Comparisons of blood lipid levels and percentages of HTG and HRC before and after a daily meal in 33 outpatients}

In 33 outpatients, non-fasting levels of TG and RC at $2 \mathrm{~h}$ after a daily meal were significantly higher than fasting levels of TG and RC ( $P<0.05$, Fig. 3A-B). According to the cut-off values of HTG and HRC recommended by the EAS [12], the percentages of HTG and HRC in the fasting state were $57.6 \%$ and $51.5 \%$, respectively, and those in the non-fasting group were $51.5 \%$ and $51.5 \%$, respectively. There was no significant difference in the percentages of HTG or HRC between fasting state and non-fasting state at 2 h after a daily meal (Fig. 3C-D).

\subsection{Awareness and acceptance of non-fasting lipid test}

For the first question about awareness of non-fasting blood lipid test, $72.7 \%$ of outpatients chose the option of 'fasting', $18.5 \%$ chose the option of 'non-fasting', no one chose the option of 'either is OK', and $9.3 \%$ chose the option of 'having no idea' (Fig. $5 \mathrm{~A}$ ).

For the second question about acceptance of non-fasting blood lipid test after immediate notification, $37.1 \%$ of outpatients chose the option of 'fasting', $10.3 \%$ chose the option of 'non-fasting', $19.6 \%$ chose the option of 'either is OK', and 33.0\% chose the option of 'depending on doctor' (Fig. 5B).

\section{Discussion}

In this study, prominently elevated levels of TG and RC were found in the non-fasting group when compared with those in the fasting one, which is similar to the finding of the studies with a large-scale general population in Denmark $[8,15,16]$. In those Danish studies, the comparison between fasting and non-fasting blood lipid levels was carried out in different subjects according to the time of visiting the clinics $[15,16]$. The significant elevation of non-fasting TG and RC levels after a daily meal was also observed in 33 fasting outpatients with HBP in this study. Moreover, the mean TG level of the fasting outpatients was more than $1.7 \mathrm{mmol} / \mathrm{L}$ and that of the non-fasting outpatients exceeded $2.0 \mathrm{mmol} / \mathrm{L}$, indicating the augmented synthesis and/or reduced elimination of TRLs and their remnants after a daily meal in Chinese patients with HBP.

Conversely, the non-fasting group had significantly lower levels of TC, LDL-C and non-HDL-C than the fasting group, which was similar to the finding of a Danish study carried out in different subjects according to the time of visiting the clinics [12]. We recently observed the non-fasting reduction in levels of TC, LDL-C and non-HDL-C in the inpatients with overweight, coronary heart disease or not [17-19]. 
There were several possible explanations for the non-fasting reduction in those lipid parameters. First, the decrease in non-fasting levels of TC, LDL-C and non-HDL-C was likely due to hemodilution caused by dietary fluid intake $[20,21]$. Second, increased TRLs and their remnants after a daily meal could activate cholesteryl ester transporter protein in the case of hypertriglyceridemia, which ultimately increased RC levels and decreased LDL-C through promoting the transfer of cholesteryl ester from LDL to TRLs and their remnants $[22,23]$. It is noteworthy that there was no significant difference in HDL-C level between the two groups, which was consistent with the results of other researchers who confirmed that HDL-C level was not affected by food intake [12].

We recently reported that the non-fasting cut-off values to determine HTG or HRC in Chinese subjects were very close to those recommended by the EAS (12), which induced that the corresponding percentages of non-fasting HTG or HRC dependent on those values were also very similar. Compared with the fasting group, the non-fasting group exhibited higher percentages of HTG and HRC. Although the difference in the percentages of HTG or HRC between the non-fasting group and the fasting one could not be statistically analyzed, the higher percentage of HTG in the non-fasting group still suggested that nonfasting blood lipid test could increase the probability of detecting HTG in Chinese outpatients with HBP.

Interestingly, the difference in the percentages of fasting and non-fasting HTG was small and insignificant in 33 outpatients with HBP. In addition to the small sample size, another cause for this phenomenon could be the difference in the time of blood lipid test. We previously found that levels of TG and $\mathrm{RC}$ began to rise at $2 \mathrm{~h}$ and peaked at $4 \mathrm{~h}$ after a daily meal in Chinese in patients with coronary heart disease and HBP $[13,14]$. For some patients who are used to a high-fat and high-calorie diet, the peak of TG level may be higher or later $[24,25]$. It indicated that serum TG level at $4 \mathrm{~h}$ after a daily meal can better reflect the maximum ability to synthesize and metabolize TG in a certain individual, while serum TG level at $2 \mathrm{~h}$ after a daily meal should be a relatively closer value to fasting level in Chinese subjects [12]. In the non-fasting group, about $44 \%$ outpatients $(n=61)$ were tested at $2 \mathrm{~h}$ after a daily breakfast, about $56 \%$ $(\mathrm{n}=78)$ were tested later than $2 \mathrm{~h}$ after a daily breakfast or even after a daily lunch. It was consistent with the finding that the mean TG level in the non-fasting group was more than $3.0 \mathrm{mmol} / \mathrm{L}$ while that at $2 \mathrm{~h}$ in 33 outpatients was about $2.5 \mathrm{mmol} / \mathrm{L}$. Moreover, it was reported that the average TG level after a daily lunch is higher than that at $2 \mathrm{~h}$ after a daily breakfast in Chinese subjects [26], which could be attributed to the additive effect of increasing TG by breakfast and lunch. Thus, TG level at $2 \mathrm{~h}$ after a daily meal may substitute its fasting value to evaluate HTG according to the non-fasting cut-off value for some special patients who are prone to hypoglycemia and physical weakness, such as diabetics, pregnant women, the elderly and children. However, in order to improve the diagnostic positive rate of HTG or to detect the maximum response of TG increase after a daily meal, blood lipid test at $4 \mathrm{~h}$ after a daily lunch could be more appropriate.

Chinese outpatients had little knowledge of the concept of non-fasting blood lipid test. Among the surveyed outpatients, less than one in five ones chose non-fasting blood lipid test. The vast majority of them chose fasting testing, which shows that this view has been widely accepted. After brief communication and notification on non-fasting blood lipid test, nearly half of them turned to accept non- 
fasting lipid test although more than one-third of them still insisted on fasting blood lipid test. It was worth noting that about one third of the surveyed outpatients left the decision to receive fasting or nonfasting blood lipid test to their doctors. That is to say, the application of non-fasting test in those subjects depends on their doctors. It indicates that the concepts of both doctors and patients must be updated through education in order to promote non-fasting blood lipid test in clinical practice in China.

There were several limitations in this study. First, the sample size was relatively small, especially compared with large-scale population studies abroad [8,9]. Second, given the crucial role of controlling LDL-C levels in the prevention of cardiovascular disease in HBP patients, non-fasting LDL-C levels also should be evaluated in HBP patients. Since the goal of LDL-C level in HBP patients depends on the corresponding cardiovascular risk determined by underlying diseases and risk factors, it will be more complex than the determination of non-fasting HTG or HRC. In the future, prospective studies about nonfasting LDL-C levels to assess the risk of cardiovascular diseases in Chinese patients with HBP and guide clinical therapeutics are needed.

\section{Conclusion}

Non-fasting blood lipid test could find more HBP outpatients with HTG in Chinese outpatients with HBP. However, the percentage of HTG at $2 \mathrm{~h}$ after a daily breakfast seemed to be close to that in the fasting state.

\section{Abbreviations}

HBP: hypertension; EAS: European Atherosclerosis Society; LDL-C: Low-density lipoprotein cholesterol; HDL-C: High-density lipoprotein cholesterol; Non-HDL-C: Nonhigh-density lipoprotein cholesterol; TC: Total cholesterol; TG: Triglyceride; RC: Remnant cholesterol; HTG: High triglyceride; HRC: High remnant cholesterol; TRLs: Triglyceride-rich lipoproteins; RLPs: remnant lipoproteins; CHD: coronary heart disease; BMI: body mass index; DM: diabetes mellitus

\section{Declarations}

\section{Funding}

The study was supported by National Natural Science Foundation of China (grant numbers 81270956,81470577$)$.

\section{Authors' contributions}

Ling Liu is the primary investigator and designer of this study. Peiliu Qu, Yingying Xie, Liling Guo, Jin Xu, Liyuan Zhu, Yangrong Tan, Yan Fu, Miao Zhang, Tie Wen participated in the design of this study. Yingying Xie, Peiliu Qu drafted the manuscript. Yingying Xie and Ling Liu revised the manuscript. All authors 
contributed to the article and approved the submitted version. All authors have accepted responsibility for the entire content of this manuscript and approved its submission.

\section{Competing Interests}

The authors declare that they have no competing interests.

\section{Availability of data and materials}

The datasets analyzed during the current study are available from the corresponding author on reasonable request.

\section{Ethics approval and consent to participate}

The study was approved by the Ethics Committee of the Second Xiangya Hospital of Central South University and informed consent was gained from all participants.

\section{Data availability statement}

The raw data supporting the conclusions of this article will be made available by the authors, without undue reservation.

\section{References}

1. Sander, G.E. and T.D. Giles, Hypertension and lipids: lipid factors in the hypertension syndrome. Curr Hypertens Rep, 2002. 4(6): p. 458-63.

2. Dolgalev, I.V., et al., Impact of hypertension, overweight, hypertriglyceridemia and their combination for mortality rate according to the results of a 27-year cohort prospective study. Kardiologiia, 2019. 59(11S): p. 44-52.

3. Ling, Y., et al., Serum triglyceride, high-density lipoprotein cholesterol, apolipoprotein B, and coronary heart disease in a Chinese population undergoing coronary angiography. J Clin Lipidol, 2017. 11(3): p. 646-656.

4. Tushuizen, M.E., et al., Postprandial lipid and apolipoprotein responses following three consecutive meals associate with liver fat content in type 2 diabetes and the metabolic syndrome. Atherosclerosis, 2010. 211(1): p. 308-14.

5. Liu, L. and S.P. Zhao, Expert consensus on the non-fasting blood lipid detection and clinical application. Zhonghua Nei Ke Za Zhi, 2021. 60(5): p. 400-405.

6. Lee, J.S., et al., Triglyceride and HDL-C Dyslipidemia and Risks of Coronary Heart Disease and Ischemic Stroke by Glycemic Dysregulation Status: The Strong Heart Study. Diabetes Care, 2017. 40(4): p. 529-537.

7. Lamarche, B., et al., Fasting insulin and apolipoprotein B levels and low-density lipoprotein particle size as risk factors for ischemic heart disease. JAMA, 1998. 279(24): p. 1955-61. 
8. Bansal, S., et al., Fasting compared with nonfasting triglycerides and risk of cardiovascular events in women. JAMA, 2007. 298(3): p. 309-16.

9. Nordestgaard, B.G., et al., Nonfasting triglycerides and risk of myocardial infarction, ischemic heart disease, and death in men and women. JAMA, 2007. 298(3): p. 299-308.

10. National guideline for hypertension management in China (2019). Zhonghua Xin Xue Guan Bing Za Zhi, 2020. 48(1): p. 10-46.

11. Whelton, P.K., et al., 2017 ACC/AHA/AAPA/ABC/ACPM/AGS/APhA/ASH/ASPC/NMA/PCNA Guideline for the Prevention, Detection, Evaluation, and Management of High Blood Pressure in Adults: A Report of the American College of Cardiology/American Heart Association Task Force on Clinical Practice Guidelines. J Am Coll Cardiol, 2018. 71(19): p. e127-e248.

12. Nordestgaard, B.G., et al., Fasting Is Not Routinely Required for Determination of a Lipid Profile: Clinical and Laboratory Implications Including Flagging at Desirable Concentration Cutpoints-A Joint Consensus Statement from the European Atherosclerosis Society and European Federation of Clinical Chemistry and Laboratory Medicine. Clin Chem, 2016. 62(7): p. 930-46.

13. Xu, J., et al., Determination of optimal cut-off points after a high-fat meal corresponding to fasting elevations of triglyceride and remnant cholesterol in Chinese subjects. Lipids Health Dis, 2019. 18(1): p. 206.

14. Xu, J., et al., Change in Postprandial Level of Remnant Cholesterol After a Daily Breakfast in Chinese Patients With Hypertension. Front Cardiovasc Med, 2021. 8: p. 685385.

15. Tushuizen, M.E., et al., Postprandial lipid and apolipoprotein responses following three consecutive meals associate with liver fat content in type 2 diabetes and the metabolic syndrome. Atherosclerosis, 2010. 211(1): p. 308-14.

16. Perez-Martinez, P., et al., Update on genetics of postprandial lipemia. Atheroscler Suppl, 2010. 11(1): p. 39-43.

17. Tian, F., et al., Changes in non-fasting concentrations of blood lipids after a daily Chinese breakfast in overweight subjects without fasting hypertriglyceridemia. Clin Chim Acta, 2019. 490: p. 147-153.

18. Guo, L.L., et al., Non-HDL-C Is More Stable Than LDL-C in Assessing the Percent Attainment of Nonfasting Lipid for Coronary Heart Disease Patients. Front Cardiovasc Med, 2021. 8: p. 649181.

19. Lin, Q., et al., The Role of Fasting LDL-C Levels in Their Non-fasting Reduction in Patients With Coronary Heart Disease. Front Cardiovasc Med, 2021. 8: p. 686234.

20. Langsted, A., J.J. Freiberg and B.G. Nordestgaard, Fasting and nonfasting lipid levels: influence of normal food intake on lipids, lipoproteins, apolipoproteins, and cardiovascular risk prediction. Circulation, 2008. 118(20): p. 2047-56.

21. Langsted, A. and B.G. Nordestgaard, Nonfasting lipids, lipoproteins, and apolipoproteins in individuals with and without diabetes: 58434 individuals from the Copenhagen General Population Study. Clin Chem, 2011. 57(3): p. 482-9.

22. Du X, S.L. Zhang and L. Liu, Association between remnant lipoprotein-cholesterol and coronary heart disease. Zhonghua Xin Xue Guan Bing Za Zhi, 2016. 44(8): p. 657-60. 
23. Mooradian, A.D., Dyslipidemia in type 2 diabetes mellitus. Nat Clin Pract Endocrinol Metab, 2009. 5(3): p. $150-9$

24. Zhao, S.P., et al., Xuezhikang, an extract of cholestin, protects endothelial function through antiinflammatory and lipid-lowering mechanisms in patients with coronary heart disease. Circulation, 2004. 110(8): p. 915-20.

25. Ling L, et al. Optimal time point to measure postprandial hypertriglyceridemia after a high-fat meal. Chin J Endocrinol Metab. 2004;(01):23-25.

26. Yang, D., et al., Postprandial Lipid Concentrations and Daytime Biological Variation of Lipids in a Healthy Chinese Population. Ann Lab Med, 2018. 38(5): p. 431-439.

\section{Figures}

A

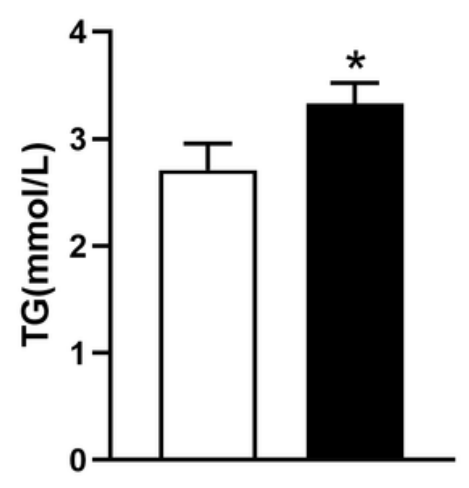

D

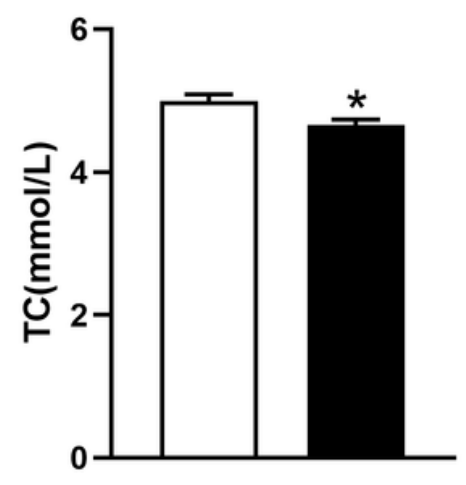

B

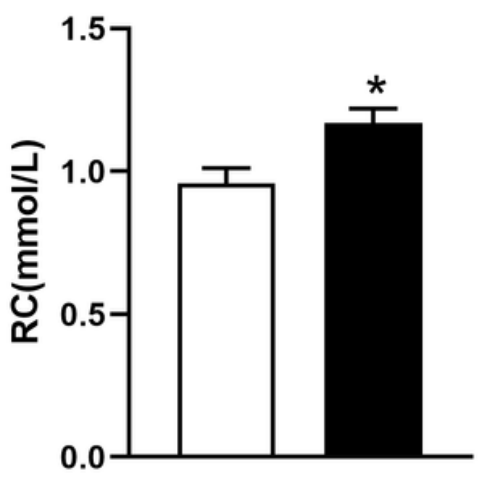

E

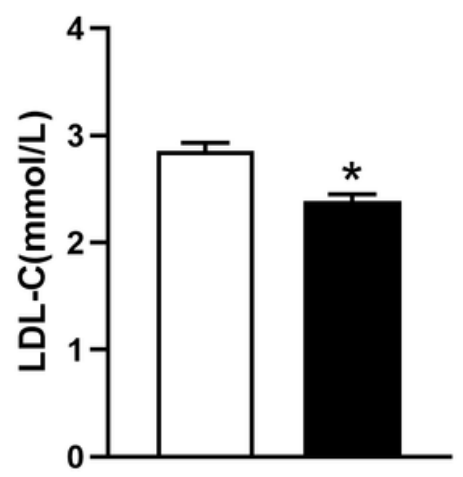

C

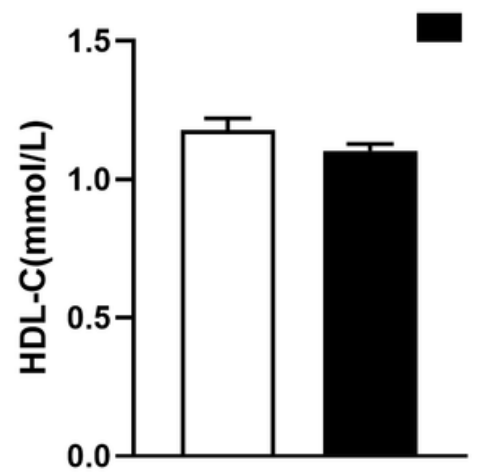

$\mathbf{F}$

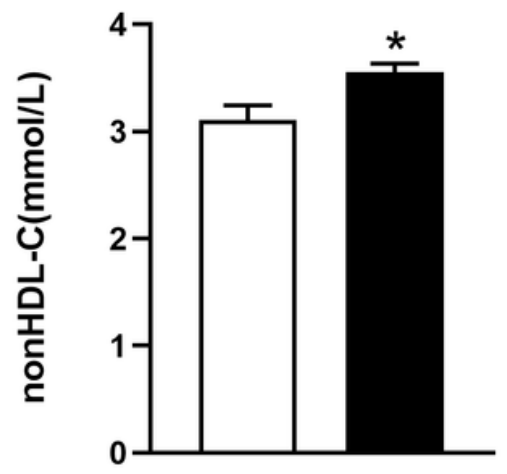

Figure 1

Comparisons of serum levels of blood lipids between the two groups. (A-F) Serum levels of TG, RC, HDL$C, T C, L D L-C$, nonHDL-C in the fasting group $(n=119)$ and non-fasting group $(n=139)$, respectively. The bar represents standard error of the mean. ${ }^{\star} \mathrm{P}<0.05$ when compared with the fasting group. 
A

$\square \mathrm{fTG} \geqslant 1.7 \mathrm{mmol} / \mathrm{L}$

$\mathrm{nfTG} \geqslant 2.0 \mathrm{mmo} / \mathrm{L}$

- $\mathrm{nfTG} \geqslant 2.02 \mathrm{mmol} / \mathrm{L}$

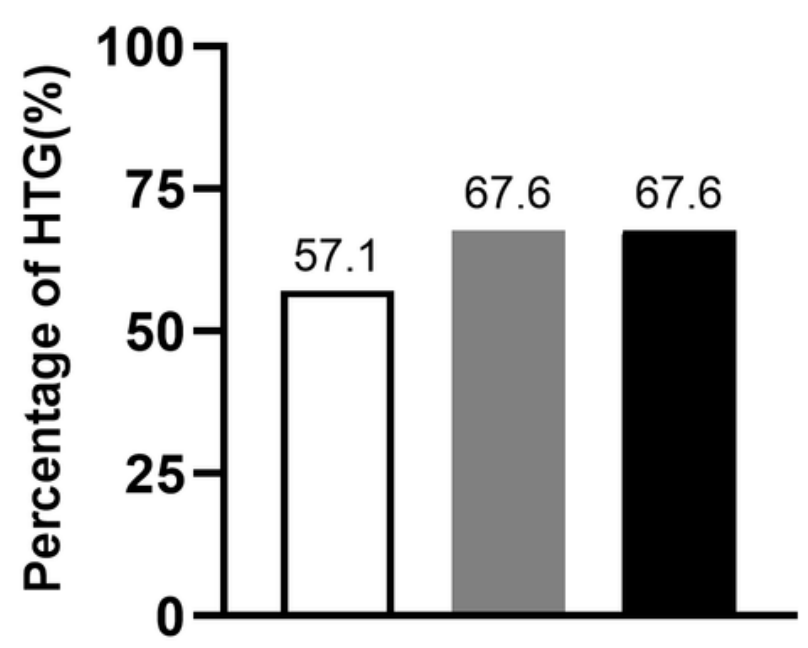

B

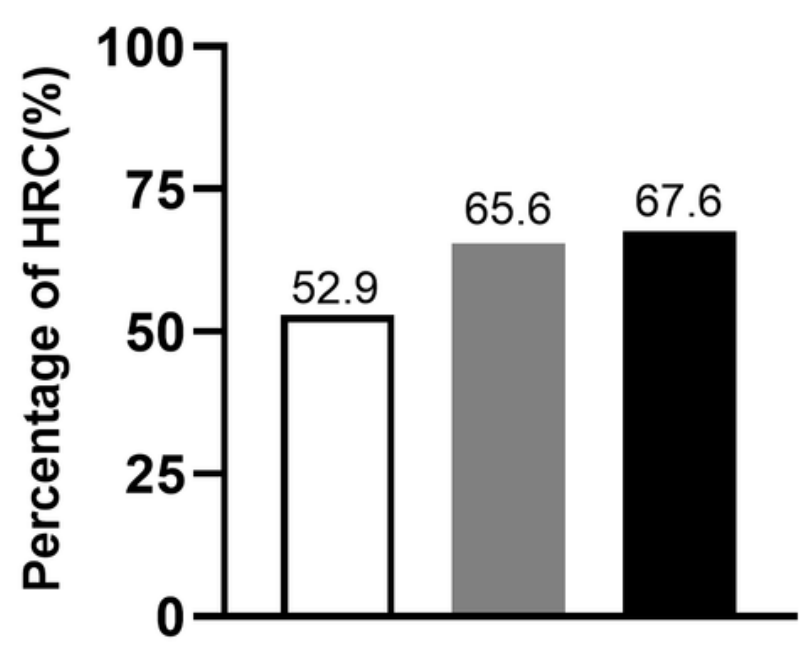

- $\mathrm{nfRC} \geqslant 0.87 \mathrm{mmol} / \mathrm{L}$

\section{Figure 2}

Comparisons of the percentages of HTG and HRC between fasting group $(n=119)$ and non-fasting group ( $n=139$ ). (A) Comparison of HTG according to the cut-off points of fasting TG (fTG) $\geq 1.7 \mathrm{mmol} / \mathrm{L}$, nonfasting $T G(n f T G) \geq 2.0 \mathrm{mmol}$ and $n f T G \geq 2.02 \mathrm{mmol} / \mathrm{L}$, respectively. (B) Comparison of HRC according to the cut-off points of fasting RC (fRC) $\geq 0.8 \mathrm{mmol} / \mathrm{L}$, non-fasting $\mathrm{RC}(\mathrm{nfRC}) \geq 0.9 \mathrm{mmol} / \mathrm{L}$ and $\mathrm{nfRC} \geq$ $0.87 \mathrm{mmol} / \mathrm{L}$, respectively. 
A

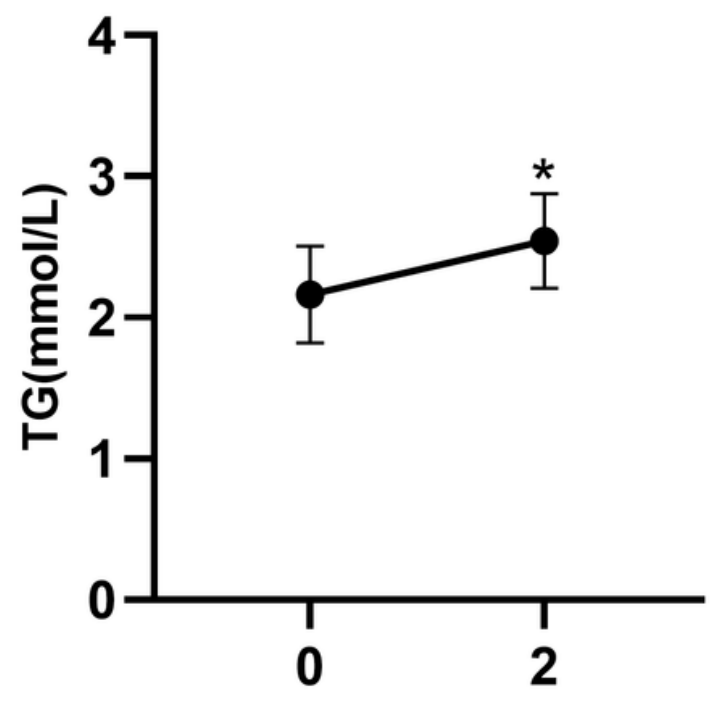

Time after meal(h)
B

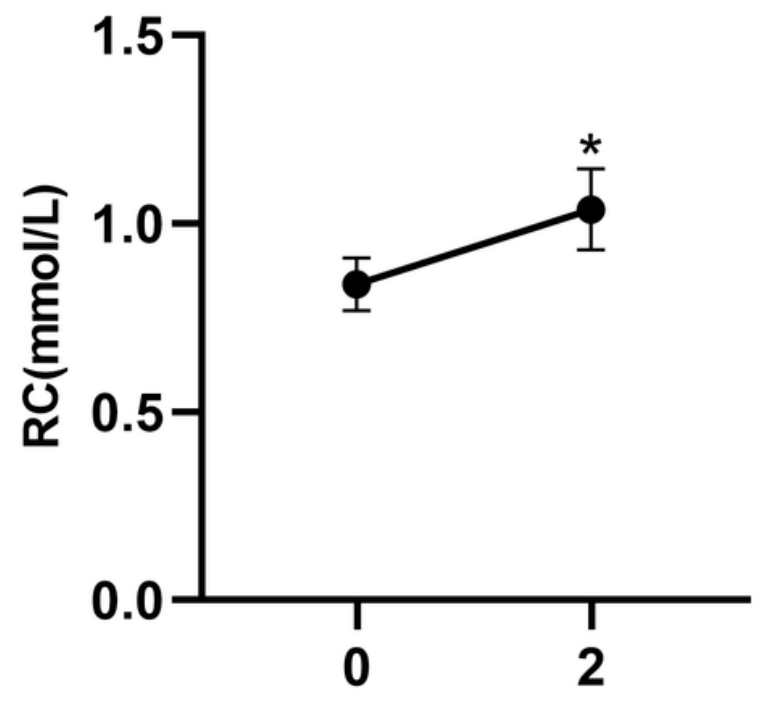

Time after meal(h)
C

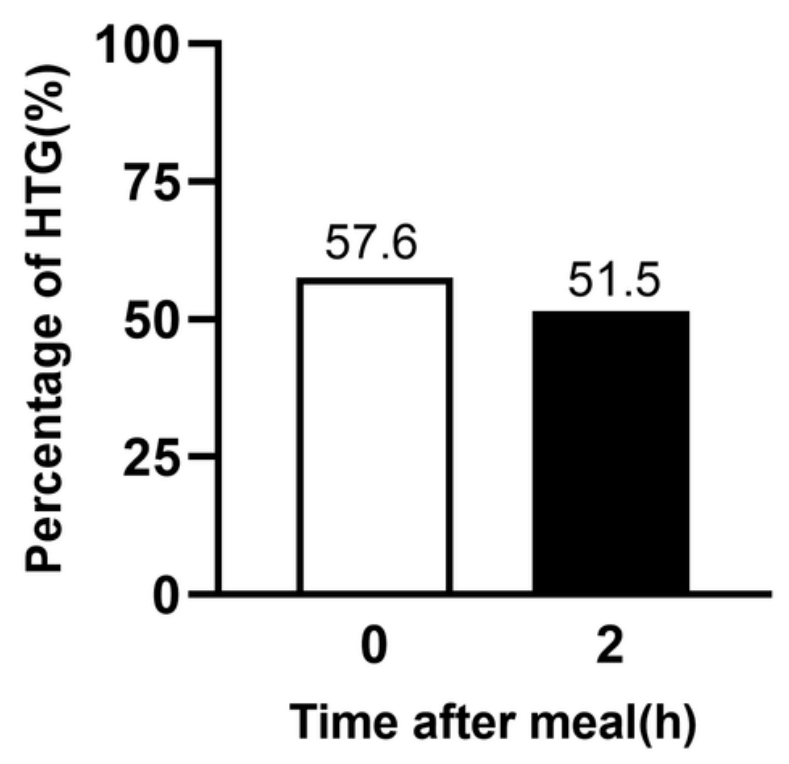

D

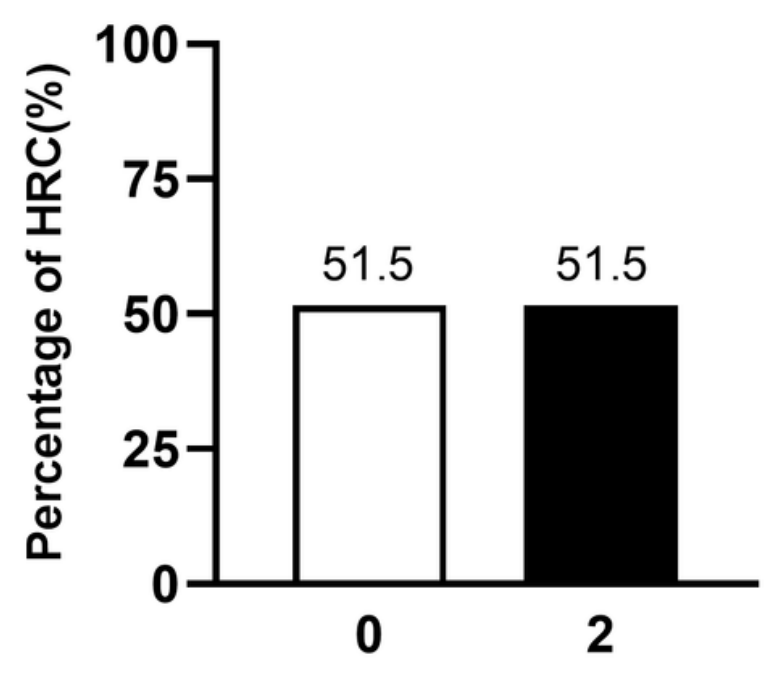

Time after meal(h)

Figure 3

Changes in serum levels of blood lipids and comparison of percentages of HTG and HRC in the outpatients with HBP ( $n=33)$. (A-B) Non-fasting changes in serum levels of TG and RC between $0 \mathrm{~h}$ and 2 $h$ after a daily meal. The bar represents standard error of the mean. (C-D) Percentages of HTG and HRC at Oh and $2 \mathrm{~h}$ after a daily meal according to $n f T G \geq 2.0 \mathrm{mmol} / \mathrm{L}$ and $\mathrm{nfRC} \geq 0.87 \mathrm{mmol} / \mathrm{L}$, respectively. 

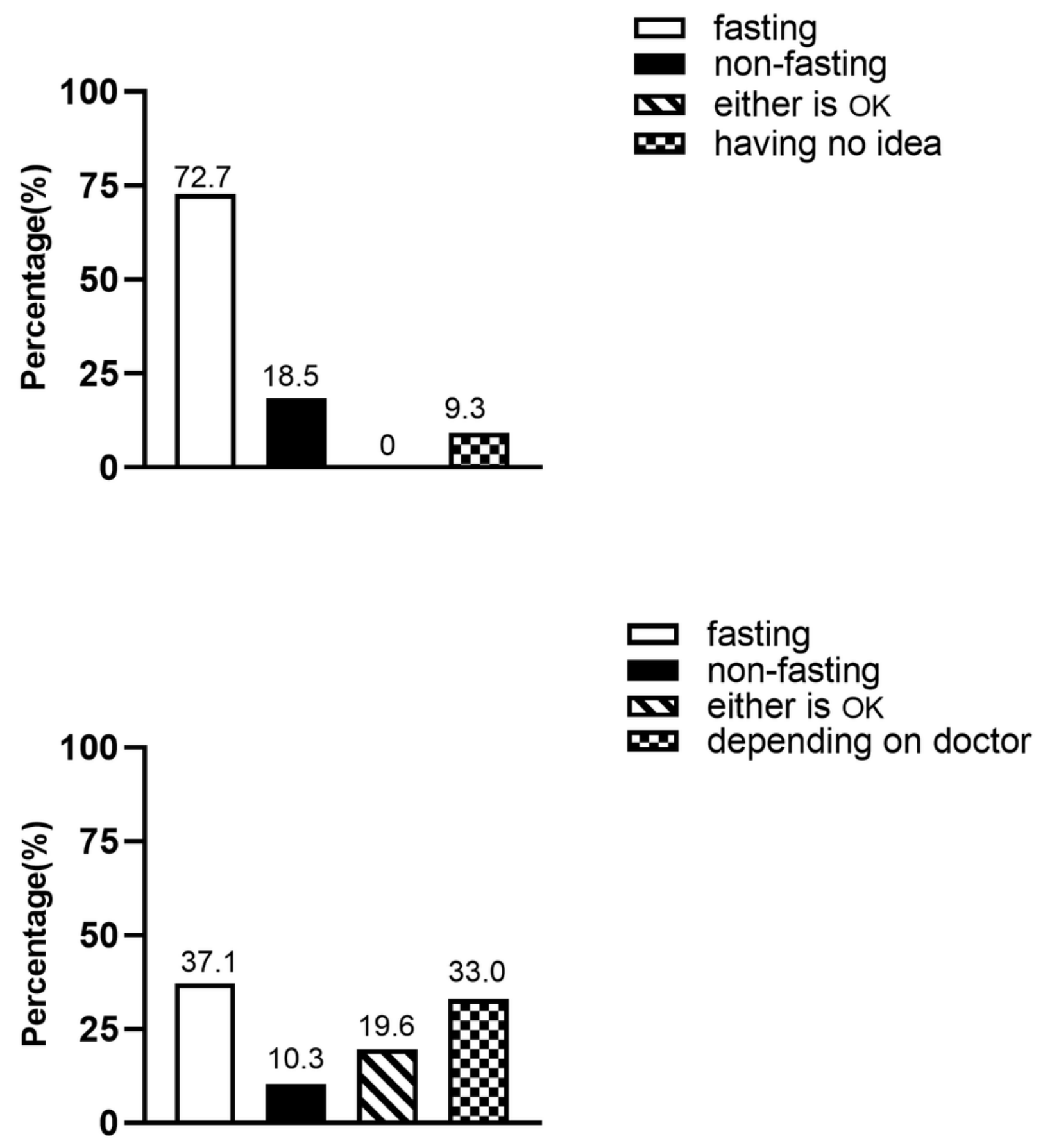

Figure 4

Awareness and acceptance of non-fasting blood lipid test. (A) Awareness of non-fasting blood lipid test in HBP outpatients. (B) Acceptance of non-fasting blood lipid test in HBP outpatients after patient education.

\section{Supplementary Files}


This is a list of supplementary files associated with this preprint. Click to download.

- originaldata.xlsx

- supplementarydata.tif 